\title{
The 'SAFE' initiative - An innovative approach to safer patient care in a tertiary hospital setting
}

\author{
Deepan Krishnasivam, Lesley Bennett, Katherine Birkett, Tim Bowles \\ Royal Perth Hospital, Perth, Western Australia
}

Received: January 3, 2019

Accepted: January 27, 2019

Online Published: January 30, 2019

DOI: $10.5430 /$ jha.v8n1p65

URL: https://doi.org/10.5430/jha.v8n1p65

\begin{abstract}
Introduction: Out of hours inpatient care within Australia, defined as the hours outside of 08:00 to 16:00 hours Monday to Friday, is traditionally provided by on-call medical teams, working in silos, supported by onsite junior medical staff. This model can be associated with poor communication both between and within teams, lack of accountability, failure of escalation, and a reactive model of care. International literature reveals that the outcomes of patients admitted to hospital out of hours are poorer, resulting in a discrepancy in mortality between in and out of hours admissions.

Methods: We aimed to reduce the discrepancy in mortality between in and out of hours admissions, as well as reducing overall inpatient mortality. Using a resilience engineering approach, we introduced a novel model of out of hours care - the "Safety After Hours for Everyone" (SAFE) Team. This incorporated a departmental model, with clear and robust internal leadership, external accountability, and formal processes for identification, review and follow up of at risk patients, as well as protocolised escalation processes.

Results: The introduction of the SAFE model has been associated with a continuous reduction in the overall Hospital Standardised Mortality Ratio (HSMR) from 0.71 to 0.54 (periods January to March 2015 vs. January to March 2018. In addition, the SAFE model has been associated with a reduction in out of hours mortality (defined as admissions from 16:00 to 08:00 hours) from 0.98 to 0.38 (periods January to March 2015 vs. January to March 2018). This has been accompanied by a qualitative improvement in the quality of care delivered out of hours, and improved satisfaction with working conditions and training delivered out of hours. Due to a drastic reduction in unplanned Resident Medical Officer (RMO) overtime associated with the introduction of the model, implementation was near cost neutral.

Conclusions: The introduction of the SAFE model has been associated with improved hospital outcomes, in conjunction with improved medical and nursing staff experiences, at a low marginal cost. This model has scope to be applied to similar tertiary level hospitals, or modified to fit within most hospital structures. A key component to the success of this model's innovation, is acknowledgement of the importance of after hours care provision to patients, highlighted by the formation of a department of after hours medicine as part of the SAFE model.
\end{abstract}

Key Words: Out of hours, mortality, after hours, junior doctors, Resident Medical Officer, 'SAFE'

\section{INTRODUCTION}

After hours inpatient care is an area of medicine that has gained increased attention in the last few years. There has been a culture of "surviving the night" overnight and over the weekend until the primary home teams return. Most Australian hospitals are staffed to provide a functional "in hours period" of 08:00 to 16:00 hours Monday to Friday (although geographical variations to this exist). Similar time

*Correspondence: Deepan Krishnasivam; Email: deepan.krishnasivam@ health.wa.gov.au; Address: Royal Perth Hospital, Perth, Western Australia. 
periods are considered as normal working hours in most other countries internationally. ${ }^{[1]}$ The rest of this time period, including weekends, is considered "after hours", often associated with significantly altered medical staffing levels and medical working practices. ${ }^{[1,2]}$

There is an internationally recognised discrepancy in outcomes noted between in and out of hours patient outcomes, with an excess mortality specifically noted with out of hours admissions. ${ }^{[1-5]}$ The literature reveals that after hours care can be suboptimal with delays to medical reviews, lack of senior clinician input in treatment decisions, completion of investigations, commencement of treatment and poor utilisation and escalation of early warning systems. ${ }^{[6-10]}$ Often in most hospitals, junior medical officers are employed after hours to manage patients. ${ }^{[1,2,9]}$ These junior medical officers (due to their clinical experience are often designated as Resident Medical Officers (RMOs) within our local Australian context) attempt to manage numerous potentially complex patients during the after hours period with limited senior doctor support and availability. ${ }^{[2]}$

This story was no different at Royal Perth Hospital (RPH). $\mathrm{RPH}$ is a 450 bedded institution, acting as the tertiary referral centre for a population of approximately 750,000 Western Australians, and is the State Major Trauma Centre. Major medical and surgical specialties are provided. Historically, out of hours care was provided by a small number of RMOs, supported by a Registrar (a slightly senior doctor who was also responsible for medical admissions), and non-resident specialist teams who were often not in the hospital, but oncall for telephone advice. Reconfiguration of health services across Western Australia in 2015 led to a reduction in on-call medical staff resulting in the historical model being unsustainable and provided the opportunity to review how medical care was delivered out of hours.

Review of the RPH Health Roundtable (HRT) data demonstrated that, consistent with international findings, RPH had a higher HSMR for after-hours admissions than in-hours admissions. ${ }^{[11]}$ In addition, adverse event reports and root cause analyses demonstrated issues with the reactive model of care, including failed handovers, poor communication and role delineation, failure to recognise and respond to altering patient circumstances, and failure to escalate deterioration appropriately. These issues continued despite implementation of recommendations from root cause analyses panels, suggesting a more coordinated and fundamental change was required.

\section{METHOD}

\subsection{Model design}

We used resilience engineering firstly to understand the "story" underlying the events, and to secondly examine why events normally do not occur. We asked genuinely curious questions of the RMOs to understand their circumstances. Major concerns surfaced; unmanageable workloads, little feedback or orientation, working occasional night shifts without education, no social or departmental support when things went wrong.

In comparison, we examined how day time clinical teams worked. Most had a departmental structure, allowing a clear feedback and governance structure. Doctors are allocated for whole training terms, allowing consistent exposure, education, and the development of a culture of performance. Clinically, the successful teams were aware of who their sickest patients were, and proactively managed them. They had a culture of appropriate delegation and escalation; and maintained closed loop feedback regarding progress.

We also identified a consistent factor where events had gone well overnight. A cadre of senior nurses existed with significant organisational experience and knowledge. Prior to implementation of the SAFE model these nurses were often working in isolation, without the resource or authority to escalate and coordinate care. However, they provided a safety net when crises arose, although unfortunately, were not always empowered to take the needed actions. Our findings were not too dissimilar from those mentioned in the literature, suggesting that this is not an issue specific to RPH, but generally applicable to most hospitals nationally and internationally. ${ }^{[1,12,13]}$

The literature reveals other out of hospital models created to address this issue internationally. These configurations also acknowledged the need for a specific focus on after hours care and a focus on improvements in communication and interactions between teams. ${ }^{[14-17]}$ Using concepts of these prior models, the SAFE model was developed to attempt to incorporate the features of successful daytime clinical teams into out of hours working, while valuing and strengthening the role of the senior nurses who had traditionally contributed to organisational resilience. The model developed allowed strong internal leadership with clear external accountability and governance, while encouraging good professional habits of communication, proactive care and appropriate delegation and escalation. The service now provides a unique, departmental approach to after-hours care to the general medical and surgical wards. A schematic of the SAFE model is outlined in Figure 1. 


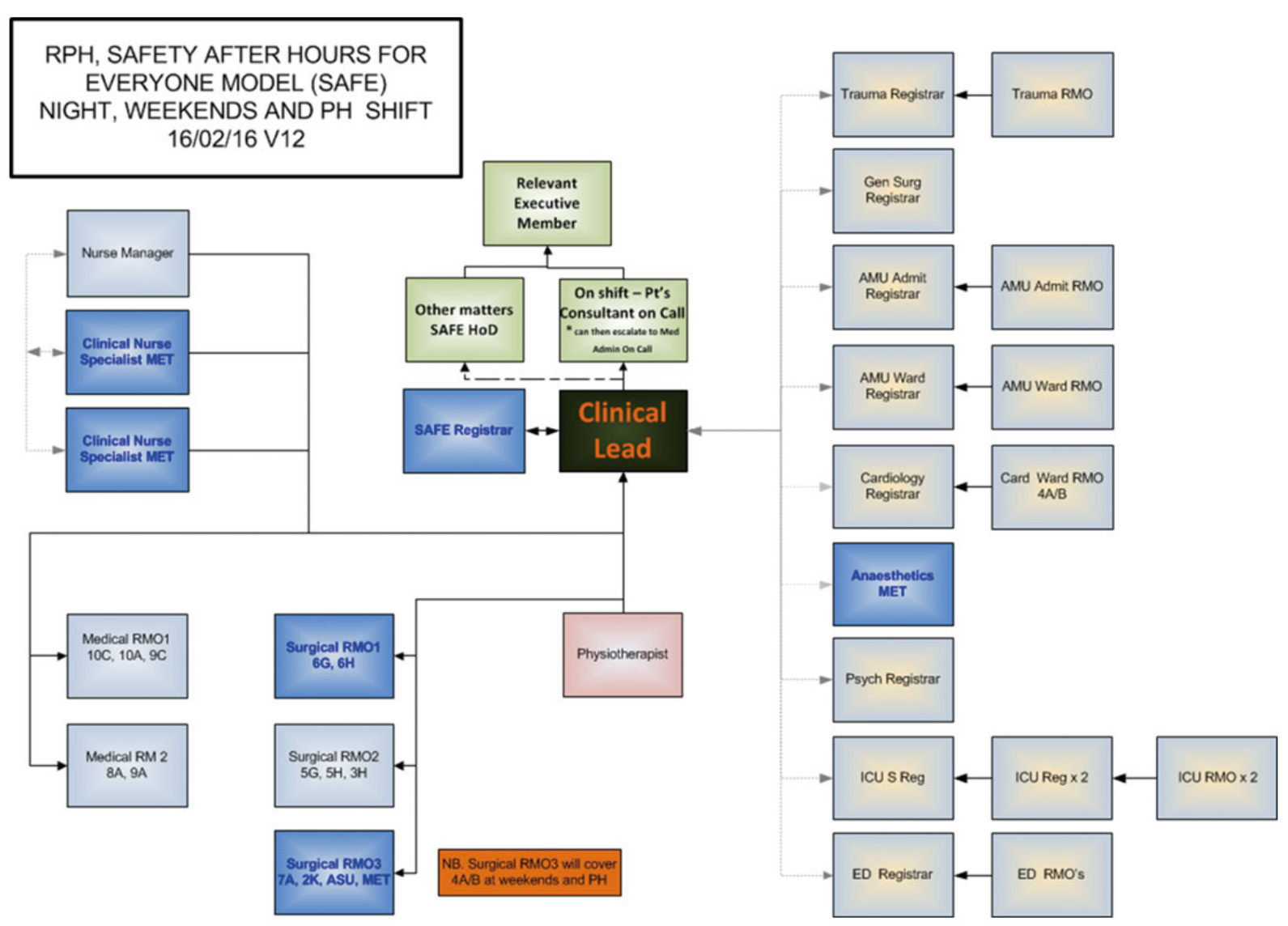

Figure 1. The 'SAFE' model

\subsection{Innovative and specific characteristics of the SAFE model}

Key innovative characteristics of the SAFE model that distinguish it from traditional after hours models are summarised below:

(1) Formation of a dedicated department responsible for out of hours inpatient care. This allows:

- Appointment of a Head of Department. This produces clear accountability for out of hours care, as well as a route to highlighting problems with out of care throughout the organisation.

- Representation of out of hours issues at senior committee meetings, maintaining the visibility of out of hours care at senior levels.

- Appointment of permanent staff, facilitating the generation of organisational memory in out of hours care, as well as the formation of an appropriate culture focusing on high- quality, safe and professional out of hours care.

- Training accreditation for out of hours terms. This allows RMOs to work in teams for whole terms.

- Internal and external governance processes including specific morbidity and mortality reviews related to care provided.

- Development of trust between in hours specialty teams and out of hours care teams.

(2) Formation of an organisation wide "Patients of Concern" list. These patients meet pre-defined criteria for high probability for deterioration, either by previous course of illness, current physiology, or specific concern from the medical or nursing team. This list provides the core patients for proactive management, in an effort to prevent deterioration.

(3) The introduction of the role of "Clinical Lead". In the SAFE model, these are Clinical Nurse Consultants in out of hours care. Their overall role as permanent staff members is to provide a stability to the team, leveraging long term experience in out of hours care, and the organisation, to predict, prevent, and resolve events. To achieve this, they take overall responsibility for the maintenance of the Patients of Concern list, with continuous closed loop communication with the medical and ward nursing staff, to confirm clinical state. There is an Escalation of Care protocol that ensures the clinical lead is empowered to escalate appropriately to Senior Medical Staff where concerns about care exist. They also act to maintain safe working conditions and appropriate 
rate of response by monitoring RMO and ward workload and dynamically task shifting as required.

(4) RMOs and Registrars are allocated for full training terms. This allows specific training in the medical problems associated with out of hours care, including the recognition and response to deterioration. Additionally, the training term allows the integration of the RMOs into the team, allowing them to adopt the culture of the unit. This has enhanced communication and optimised professional exposure to a wide variety of clinical settings.

(5) RMOs are allocated to specific wards, rather than clinical specialties. Clear communication with senior nursing staff on the wards is mandated, such that the RMOs feel part of the ward team as well as the SAFE team. All patients entering the RMO's clinical area for any reason are reviewed, generating a sense of ownership and responsibility that is absent in traditional models.

(6) The shift commences with a formal handover, centered on a "Patients of Concern" list. The outcome of this is a shared understanding of the location of patients who are likely to deteriorate, and a mutual understanding of who has responsibility to review and monitor these patients. It also builds a shared understanding of areas of particular workload concern, allowing work to be redistributed as needed.

(7) Strong integration of the Medical Emergency Team (MET) service into the out of hours care model, recognising that emergency response to deteriorating patients is a core role of the out of hours service. Different terms may be used internationally, but in essence, the MET team (in a local Australian context) functions as a critical care response team within the hospital setting for deteriorating patients. These patients are acutely stabilised by the MET team, and then transferred to a critical care environment such as intensive care. This is often a high stress scenario requiring senior clinician expertise. The SAFE registrar and Clinical Lead are the MET medical and nursing leaders. Given their exclusive rostering in the area, this allows the registrars to build significant experience rapidly, while drawing on the long term experience of the Clinical Leads.

(8) A very high importance is placed on welfare, recognising that allocation of RMOs to 10 -week terms of out of hours care can be socially and professionally isolating. Efforts are made to accommodate significant shift requests. The RMOs are allocated to teams which they retain for the whole term, allowing supportive relationships. Debriefing and welfare checks are prioritised, as are external sources of support.

\subsection{Implementation}

The model was introduced in January, 2016. The departmental framework was developed in mid 2015, to provide a policy and administrative framework on launch. Prior to launch, broad engagement with multi-level staff groups occurred to ensure successful implementation. These sessions focused on areas of interaction, such as handover methods, task requesting, and formation of the Patient of Concern list. This was combined with focused nursing education to ensure that all relevant ward areas were familiar with the program at launch. The launch was timed to coincide with RMO handover. Due to staggered handover dates, the team were supported by experienced registrars for the first month of the rotation.

\subsection{Analysis and measurement methods}

Core measures of success and assessment methods, included:

- Overall Hospital Standardised Mortality Rate (HSMR), collected from Health Roundtable (HRT) Data

- Ratio of mortality for in and out of hours admissions, again collected from HRT HSMR data

- Staff satisfaction with the care provided

\section{RESULTS}

\subsection{Hospital standardised mortality ratio}

Latest data from Health Round table has shown an improvement in mortality, with a fall in global HSMR from 0.71 in the period January to March 2015 (before SAFE implementation) to 0.54 January to March 2018, once the model was well established. HSMR data sourced from HRT is outlined in Figure 2. The trend of the HSMR data shows a continuous downward trend in mortality from 0.71 to 0.54 .

With an HSMR of 0.54, RPH has among the lowest standardised mortalities in Australia, reflecting the emphasis on safety and enhanced capabilities associated with the model. International literature demonstrates that admissions out of hours are associated with increased standardised mortality, and addressing this was a key goal of the SAFE model. Although there are multiple confounding factors in this association, such as improved coding and change in staffing recruitment, the implementation of the SAFE model has been the biggest operational change at RPH in the last 5 years and a sub analysis of after-hours mortality admissions further supports the contribution SAFE has made to this overall reduction. This analysis showed that the HSMR for out of hours admissions has fallen from 0.98 (January to March 2015, pre-SAFE implementation) to 0.38 (January to March 2018, most recent data available). This change in after hours mortality exceeds the overall improvement in mortality, suggesting that the SAFE model and the resultant changes in after hours care is key to the overall improvement in HSMR. These findings are also consistent with the nature of the service provided by the SAFE model.

ISSN 1927-6990 E-ISSN 1927-7008 


\section{RPH_WA's Hospital Diagnosis Standardised Mortality Ratio for the 12 months to March 2018 was 54}

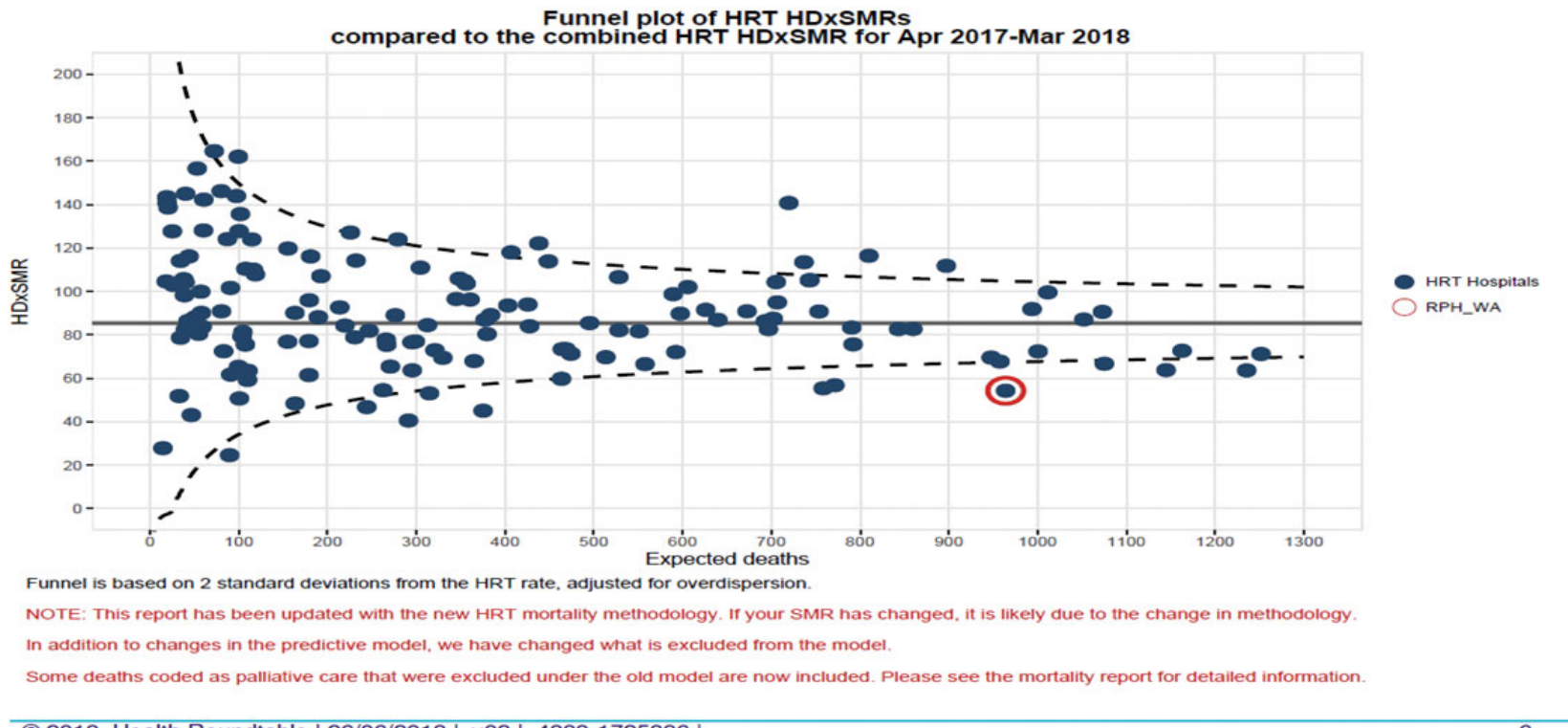

Figure 2. Funnelplot HSMR Data

\subsection{Staff feedback}

As highlighted, another key component of the model is staff feedback. Staff feedback is important in monitoring adverse trends, and to promote key safety culture concepts found in high reliability organisation.

The key findings for Staff Surveys conducted locally over the implementation period of the model is summarised below:

(1) RMOs describe a significantly improved overall experience of out of hours work. Particular positives included increased senior support and facilitation of senior review with improved perceived patient care. Time management and workload distribution were better, with improved ability to take breaks.

(2) Nursing staff feedback had identified improved confidence in the medical team after hours. It is now easier to identify the correct doctor to call, with faster and more reliable attendance. They had more confidence in the presence of a system to facilitate senior review of deteriorating patients. The presence of the Clinical Lead as a single point of contact in the event of uncertainty or conflict was highly rated. Nursing staff also rated perceived patient care as far improved after implementation of the SAFE model.

(3) There has been an overall reduction in the time to review sick patients and in the task response and completion times.
Staff satisfaction with the new model was very positive, particularly in relation to ward based RMOs, increased senior support and reduced response times to requesting patient reviews. This positive feedback and improved workplace satisfaction is key to promoting a safety culture. ${ }^{[15]}$ In addition, the SAFE model has received accreditation from the local post-medical accreditation and training council as a training position for RMOs in response to this positive feedback and structured approach to the management of critically deteriorating patients. This reinforces the staff perception of the positive impact of the SAFE model and approach to management of patient care within the hospital.

\section{Discussion}

The key success of the SAFE model within this tertiary hospital setting can be attributed to its focus on the following key areas (which can be applicable to any healthcare organisation).

\subsection{A departmental approach to after-hours care}

The key concept of this model is to provide after-hours care within a departmental framework, similar to provision of care during the in-hours space. In order to achieve this, the department was created with a dedicated Head of Department. The department commenced after-hours clinical care in January 2016 with a team made up of ward based RMOs allocated for a full term (10 weeks), a Registrar (senior doctor), Senior Nurses and a Physiotherapist, as outlined in Figure 1. The 
team is led by a Clinical Lead who coordinates, progresses and escalates care, ensuring patients receive safe and timely care. The Clinical Lead, currently a senior nurse, oversees RMOs, receives closed loop feedback on all patients of concern, and as permanent staff, provides stability of the model.

The allocation of staff to a department model provides structure and allows for clinical governance and accountability. This also allows for clear role delineation within the team, and progressive steps in terms of escalation and care provision. The Clinical Lead and the Registrar lead the service, by having appropriate medical and nursing input into patient care provision, and having supervision over RMOs. Prior to this approach, RMOs would be left to review patients without direct oversight or feedback, leading to variations in standards of care and clinical practice.

Allocation of RMOs to a ward (rather than specialty) improves continuity of care and knowledge of patients, with enhanced communications with ward nursing staff. There is increased professional exposure to a wide variety of clinical settings, and very positive feedback on both the level of supervision and teaching from RMOs who are allocated SAFE for the term of out of hours work. Feedback from nursing staff has been positive with more reliable availability of medical staff leading to development of healthy professional relationships between medical and nursing staff out of hours

The use of a departmental approach also allows for stability and cultural development. The Clinical Lead and the Registrar roles in the SAFE model are designed to be long term appointments. This appointment is through a rigorous selection process, allowing recruitment of individuals with an interest in high quality and sustainable out of hours care. The role of the SAFE Clinical Lead and Registrar is highly valued within the organisation. In addition, the Head of Department, with a primarily administrative role, allows for clear accountability and interaction between the SAFE service, other clinical teams, and executive.

Residents are given 10-week terms in the SAFE model, as opposed to working "ad-hoc" shifts over a month as in the previous model. By working training terms, residents in the team are given value and identity, and take on the team's culture. This also provides teaching and training opportunities, as well as being able to emphasise and learn best practice clinical guidelines. In addition, stress and other key components of clinician engagement, such as burnout, fatigue and increased error rates in care provision are minimised, through a team-based approach. In particular, working hours are also improved, as the out of hours shifts are fully rostered, rather than overtime in addition to normal working hours.
The departmental approach thus lends itself to providing structure in after hours care, with emphasis on patient and staff welfare. This SAFE model represents the only departmental approach to the author's knowledge in the literature at the time of writing.

\subsection{A proactive approach and the safety culture}

The other key mantra within the SAFE model is that of a proactive approach to care than a reactive approach. In order to achieve this, this model prioritises handover as key in generating a positive, proactive safety culture. Handover is centred on "Patients of Concern" meeting agreed criteria. The SAFE Team proactively reviews all identified patients with closed loop feedback to the Clinical Lead ensuring care is monitored and progressed. The model aims to progress care with a clear process of escalation. There is an emphasis on daily education with support for staff in recognising and reporting deteriorating patients earlier.

It is well realised in other big industries that Safety Culture is of key importance in improving staff and consumer welfare and improve outcomes. Indeed, the choice of the acronym SAFE reflects our prioritisation of a "safety culture" within the organisation. ${ }^{[15]}$ The aim is to emphasise the concept of safety and performance into all actions, and in fact enable staff to have a proactive approach to patient care, and associated empowerment with delivering high quality care. ${ }^{[18]}$

Other initiatives introduced simultaneously were ward board meetings, regular RMO ward rounding, and completion of "safety checks" on all patients transferring into SAFE clinical areas from other areas of the hospital, such as the operating room recovery or Emergency Department. Regular formal and informal meetings through the shift allow maintenance of team knowledge of workloads and progress of patients of concern. Non urgent tasks are requested at ward level by the nurse, with the time, urgency, patient name, bed number, name of staff member requesting and the nature of the task documented in the task folder. Pagers are now used for escalating care rather than for routine work, allowing for fewer interruptions to work and better concentration.

\subsection{Applicability to other settings}

The SAFE Model can be adapted to other settings as a strategy to address local issues and root causes identified from working after hours. The model has a number of different interconnected components that, when supported by Executive and Clinical Leadership, can influence cultural change from an ethos of survival at night to one of progression of care. Improving patient outcomes for patients in hospital after hours is a universal challenge that can be tackled by innovative solutions within a culture of continuous quality 
improvement. ${ }^{[19]}$

This model can be easily modified and adapted to other hospitals as an initiative to improve care after hours. Investment in leadership and commitment is required for successful implementation as is consistency in momentum. We have encountered many challenges prior to and during the implementation of this model. The result is that SAFE has established a precedent to which other hospitals refer. The SAFE model has had numerous benefits, and local hospitals have shown interest in adapting the model.

\section{Closing SUMMary}

The SAFE model represents a significant change in after hours working, as it highlights and reinforces the importance of after-hours care provision, and the impact of a coordinated proactive approach in improving outcomes. The reduction of in-hours and after-hours mortality is a strong testament its effectiveness. This model also fosters trusting working relationships between SAFE and the home team, which is well recognised as a key factor to improved patient care. ${ }^{[1,15,18]}$

The most exciting prospect of this model is that we have the opportunity to nurture and grow the model and to widen its scope. This signifies the first step for the members involved in the SAFE initiative to consider the transformation of this hospital into a "true" 24-hour hospital, where there is no clear distinction in terms of care between in-hours and after-hours care. The SAFE model represents a large component of this by reducing the gap between in and after-hours care, which ultimately in the end leads to better patient outcomes, the goal for all healthcare organisations.

\section{ACKNOWLEDGeMents}

The authors would like to acknowledge the executive, medical, nursing and all staff of Royal Perth Hospital. In addition, the following individuals are especially acknowledged for their time, commitment and dedication with regards to the SAFE model; Dr Aresh Anwar, Professor Grant Waterer, Ms Linda Brearley, Ms Kelly-Ann Hahn, Mr Ian Atherton, Mr Robert Wilson, Ms Elizabeth Phillips, Ms Caroline Hale, Ms Cecilia Hamukale, Mr Douglas Doust, Ms Christine Taylor, Ms Katherine Stibi, Mr Robert Lethaby, Ms Cathyrn Rhodes, Ms Joanne Cunningham, Ms Annette Baker, Ms Rebecca Crellin, Mr Terry Jongen, Ms Suzanne Martin, Ms Katie McKenzie, Dr Munish Kumar, Dr Andrew Piercy, Dr Mehkala Nanthavarman, Dr Wei Sern Tan, Dr Hairul Bin Abdul Rahman, Dr Timothy Whitmore, Dr Karen Quinlan, Dr Emily Clarke and Dr Benedict Tan.

\section{CONFLiCTS OF INTEREST Disclosure}

The authors declare they have no conflicts of interest.

\section{REFERENCES}

[1] Ruiz M, Bottle A, Aylin PP. The Global Comparators project: international comparison of 30-day in-hospital mortality by day of the week. British Medical Journal Quality Safety. 2015: 1-13. https://doi.org/101136/bmjqs-2014-003467

[2] Freemantle N, Richardson M, Wood J, et al. Weekend Hospitalization and additional risk of death: analysis of inpatient data. Journal Royal Society of Medicine. 2012; 105: 74-84. PMid:22307037. https://doi.org/10.1258/jrsm.2012.120009

[3] Aylin PP, Yunus A, Bottle A, et al. Weekend mortality for emergency admissions. A large, multicentre study. Quality and Safety in Health Care. 2010; 19(213): e217. https://doi.org/10.1136/qshc.2 008.028639

[4] Barba R, Losa JE, Velasco C, et al. Mortality among adult patients admitted to the hospital on weekends. European Journal of Internal Medicine. 2006; (17): 322-324.

[5] Bell CM, Redelmeier DA. Mortality among patients admitted to hospitals on weekends as compared with weekdays. New England Journal of Medicine. 2001; 345(9): 663-668. PMid:11547721. https://doi.org/10.1056/NEJMsa003376

[6] Seward E, Greig E, Preston S, et al. A confidential study of deaths after emergency medical admission: issues related to quality of care. Clinical Medicine. 2003; 3: 425-434: https ://doi .org/10.786 1/clinmedicine3-5-425

[7] Griffiths P, Saucedo AR, Schmidt P, et al. Vital signs monitoring in hospitals at night. Nursing Times. 2015; 111(36/37): 16-17.
[8] Gordon CL, Beckett DJ. Significant deficiencies in the overnight use of a Standardised Early Warning Scoring system in a teaching hospital. Scottish Medical Journal. 2011; 56(1): 15-18. PMid:21515526. https://doi.org/10.1258/smj.2010.010009

[9] Fossum M, Hewitt N, Weir-Phyland J, et al. Providing timely quality care after-hours: Perceptions of a hospital model of care. Collegian 2018: 2-7. https://doi.org/10.1016/j. colegn.2018.02.0 04

[10] Quirke S, Coombs M. Suboptimal care of the acutely unwell ward patient: a concept analysis. Journal Advanced Nursing. 2011; 67(8): 1834-1845. PMid:21545636. https://doi.org/10.1111/j.13 $65-2648.2011 .05664$. $x$

[11] Health Roundtable. Health Roundtable [Internet]. 2018 [cited 2018 Available from: http://home.healthroundtable.org

[12] Handel AE, Patel SV, Skingsley A, et al. Weekend admissions as an independent predictor of mortality: an analysis of Scottish hospital admissions. British Medical Journal Open. 2012; 2: e001789. https://doi.org/10.1136/bmjopen-2012-001789

[13] Sundarajan K, Flabouris A, Thompson C. Diurnal variation in the performance of rapid response systems: The role of critical care services-a review article. Journal of Intensive Care. 2016; 4(1): 1-11. PMid:26734467.

[14] Beckett D, Gordon C, Paterson R, et al. Improvement in out-of-hours outcomes following the implementation of Hospital at Night. Quarterly Journal of Medicine. 2009; 102: 539-546. PMid:19465374. https://doi.org/10.1093/qjmed/hcp056 
[15] Braithwaithe J. The Importance of Culture in Health Care Settings [and its relationship to safety and quality]. Australian Institute of Health Innovation; 2014. Available from: http://www . apha.org.au/wp-content/uploads/2014/03 /Jeffrey-Braithwaite.pdf

[16] Hamilton-Fairley D, Coakley J, Moss F. Hospital at night: An organizational design that provides safer care at night. BMC Medical Education. 2014; 14(1): S17. PMid:25561063.
[17] Singer S, Lin S, Falwell A, et al. Relationship of safety climate and safety performance in hospitals. Health Serv Res. 2009; (44): 399-421.

[18] Berwick D. Improvement, Trust and the Healthcare Workforce. Quality and Safety in Health Care. 2003; 12: 2-6. https://doi.org/ 10.1136/qhc.12.suppl_1.i2

[19] The Advisory Board Company. Hospitalists - the New Care Leaders. Australian Research Note - International Clinical Operations Board. 2015. 